\title{
Matching to successive samples: A multiple-unit memory task with rhesus monkeys
}

\author{
JAMES V. DEVINE and LUTHER C. JONES \\ The University of Texas at El Paso, El Paso, Texas 79968
}

\begin{abstract}
The delayed matching-to-sample task was extended to a multiple-unit memory procedure for nonhuman primates by increasing the number of samples shown in succession prior to the matching test. Rhesus monkeys were capable of reconstructing the order of presentation of three successively presented samples. The task is seen as an animal analogue of human memory tasks.
\end{abstract}

Studies of short-term memory (STM) with nonhuman primates have sometimes involved a procedure thought to parallel closely STM tasks used with humans, the delayed matching-to-sample (DMS) task (Jarrard \& Moise. 1971). In the typical nonspatial DMS task. a sample stimulus is exposed for the subject's inspection. the sample is removed, and a delay interval is introduced. Then two or more test stimuli. one of which matches the original sample, are presented for the subject's choice. The matching performance of monkeys after no delay is usually quite high ( $95 \%$ or better). But the nonspatial DMS studies have failed to show the rapid decrease in retention over an 18-sec delay interval reported with humans (e.g.. Peterson \& Peterson. 1959). In fact, a number of nonspatial DMS studies report above-chance performance with relatively long delay intervals: $60 \mathrm{sec}$ (Jarrard \& Moise, 1970), $232 \mathrm{sec}$ (Mello. 1971), and $120 \mathrm{sec}$ to $4 \mathrm{~min}$ (D'Amato \& O'Neill. 1971).

In DMS studies, the item to be remembered has typically been a single overlearned color or shape: in human memory studies, the item has been a multiple unit. e.g. three consonants. We have designed a memory task for nonhuman primates that involves the retention of a multiple unit. A variation of the simple matching-to-sample task was used, matching to successive samples (MSS). In its simplest form, i.e., two successive samples with two test stimuli (2-2). a single sample is shown to the subject, then removed from view, and a second sample is briefly presented. After a delay interval, the two previously shown stimuli are presented. The subject indicates (reconstructs) the order of presentation of the samples by selecting the first and then the second sample. If

This research was supported by The University of Texas at E! Paso Research Institute. the 6571st Aeromedical Division, and Albany Medical College. Holloman A.F.B. We thank Don Farrer for the loan of the apparatus and Judith Goggin for comments on the inanuscript. Requests for reprints should be sent to James $V$. Devine. Department of Psychology. University of Texas at El Paso. El Paso. Texas 79968. L. C. Jones is now at University of New South Wales. Australia 2033. the subject selects the second sample as his first choice, he is incorrect.

Since the 2-2 task may be solved by attending to one sample. we extended the MSS task to two more complex variations: two successive samples with three choice stimuli (2-3) and three successive samples with three choice stimuli (3-3). Both variations required observation of at least two successive samples. They differ in that the 2-3 task involves both discrimination of the two samples from a negative stimulus and reconstruction of the order of presentation; the 3-3 task requires only reconstruction of the order of the sample set. Since little was known concerning the training required for establishing successive matching-to-sample, our study was designed to investigate (a) the rhesus monkey's competence in the various tasks, and (b) the training techniques that facilitate acquisition of the tasks.

\section{METHOD}

\begin{abstract}
Subjects
Two experimentally naive, adolsecent male rhesus monkeys (.Macacu mulatra). weighing approximately $4.3 \mathrm{~kg}$. were maintained at $85^{\circ}{ }_{0}$ to $90^{\circ \%}$ of their pre-experimental bods weights throughout the experiment.
\end{abstract}

\begin{abstract}
Apparatus
The subjects were unrestrained in a large primate test chamber $1.914 \mathrm{~m}$ long. $.762 \mathrm{~m}$ wide, and $1.524 \mathrm{~m}$ high, with a plywood floor) similar to that used by Rohles (1961). The steel-framed chamber contained a horizontal row of 12 in-line digital displays and a sample display positioned $68 \mathrm{~mm}$ above the sixth display. The displays were designed to project stimuli from behind Plexiglas disks $138.10 \mathrm{~mm}$ in diam and $25.4 \mathrm{~mm}$ apart). Microswitches recorded and defined a response when 1 of the 12 comparison disks was pressed. On! 2 of the 12 comparison disks ( 5 th and 6 th from the subjects left) were used in the $1-1.1 \cdot 2$. and 2.2 tasks. The 7 th disk from the subjects" left was introduced for the 2-3 and 3-3 tasks. Responses to the sample disk did not activate the recording or programming equipment.

The entire display system and $190 \mathrm{mg}$ banana-pellet dispenser were programmed by a HERCO Matching-to-Successive Samples Device (a hard-wired system constructed by Houston Engineering and Research $C_{0}$.) in conjunction with a Friden tape-driven programmatic Flexowriter. Program tapes controlled stimulus presentation and position. Sample duration, separation between
\end{abstract}


Table 1

Mean Number of Training Trials and Level of Performance on the Last 100 Trials at Each Step of Training

\begin{tabular}{cccc}
\hline & Task & & \\
\hline Samples & $\begin{array}{c}\text { Test } \\
\text { Stimuli }\end{array}$ & $\begin{array}{c}\text { Mean } \\
\text { Trials }\end{array}$ & $\begin{array}{c}\text { Percent } \\
\text { Correct }\end{array}$ \\
\hline 1 & 1 & 351 & 97 \\
1 & 2 & 2780 & 91 \\
2 & 2 & 3222 & 85 \\
2 & 3 & 1686 & 50 \\
3 & 3 & 1517 & 65 \\
\hline
\end{tabular}

samples. separation between the last sample and choices (delay interval), and intertrial interval (ITI) were manually controlled.

\section{Stimuli}

Ten two-dimensional stimuli were used: five solid white shapes on a black background (triangle, $X$, square, horizontal line, and vertical line) and five colors covering the entire disk (red, green, yellow, blue, and white).

\section{Procedure}

Five matching-to-sample tasks of increasing complexity were studied. Task complexity was increased by increasing the number of samples in the sample set (one to three samples) or the number of choices in the test set fone to three test stimuli). For example, after testing on the simple one-sample/one-test-stimulus task (1.1), we presented a 1-2, then a 2-2 task, etc. (see Column 1 of Table 1). The majority of trials were arranged so that the 10 stimuli were presented an equal number of times as test stimuli (samples) in each response disk position. If position or stimulus preferences developed, trials were massed against the preference until it was eliminated. This was done in order to facilitate acquisition of the problem and was necessary only in the early stages of training.

In the 1-1 training. a sample stimulus was exposed for $2 \mathrm{sec}$, followed within $30 \mathrm{msec}$ by the same stimulus on one of the two response disks. (Our apparatus did not allow simultaneous matching, and $30 \mathrm{msec}$ separation between samples was the minimum possible.) Responses to the choice disk were reinforced, while responses to an unlit disk aborted the trial. ITIs of $10 \mathrm{sec}$ for a correct response and $16 \mathrm{sec}$ for an incorrect response were used during $1-1$ task training and in the majority of the tasks, with noted exceptions. The subjects were placed in the test chamber for $30 \mathrm{~min}$ or for 60 trials. whichever came first. Training continued until the animals responded within $2 \mathrm{sec}$ to the correct-choice disk and were correct in $90 \%$ of their choices for 2 consecutive days.

The 1-2 task was initiated with a 10-sec sample exposure, gradually decreased to $2 \mathrm{sec}$ using a $3: 3$ titration procedure (i.e., three consecutive correct responses to decrease exposure time $1 \mathrm{sec}$ and three consecutive incorrect responses to increase exposure time $1 \mathrm{sec})$. The monkeys were trained on the $1-2$ task until they responded at $90 \%$ correct for 2 days of 100 trials/day.

Because the subjects were allowed to move freely in the test apparatus. it was considered desirable to determine whether or not the animals could match with brief sample durations. After meeting criterion on the 1-2 task, the monkeys were given 2 days of decreasing sample duration training. Both animals were capable of matching to samples exposed for approximately $30 \mathrm{msec}$.

In order to ease the transition from single-sample problems to two-successive-samples problems, the first sample in the 2-2 task was exposed for $2 \mathrm{sec}$ and the second sample for $.50 \mathrm{sec}$. Then the second sample's exposure duration was gradually increased on the basis of a $3: 3$ titration ratio in $.10-$ sec steps until it was also exposed for $2 \mathrm{sec}$. The time interval between successive samples and between the termination of the second sample and the choices was $30 \mathrm{msec}$. The number of training trials/day varied between 80 and 100; over 3.000 total trials of $2-2$ training were given to each subject. The 2.2 task training was followed by a titration experiment in which the sample duration for both samples was decreased in $.10-\mathrm{sec}$ steps from $2-\mathrm{sec}$ exposure time to $300 \mathrm{msec}$. Two days were devoted to sample-duration titration of 100 trials day.

In the 2-3 and 3-3 tasks, the first. second, and third sample (or distractor in the 2-3 task) appeared equally often on the three response disks. The six possible sample/response-disk arrangements were balanced in blocks of 48 trials. Also, we arranged the test stimuli combinations within blocks of 48 trials: 12 trials of three shapes, 12 trials of two colors and one shape, etc. A more complete balance would involve equal presentation of the eight possible combinations.

The 3-3 task training began with the first two samples exposed for $2 \mathrm{sec}$ each and the third sample for $30 \mathrm{msec}$. A 3:3 titration procedure with a step of approximately .10 to $.20 \mathrm{sec}$ was used for the third sample. ITI was increased to $30 \mathrm{sec}$ for a correct response and $45 \mathrm{sec}$ for an incorrect response. The separation between samples and the interval from the termination of the last sample to the presentation of the choices was approximately $30 \mathrm{msec}$.

\section{RESULTS AND DISCUSSION}

As the number of elements of the sample set and/or the test set increased, the difficulty of the task increased. As shown in Table 1, multiple-unit tasks were within the capability of the monkeys. After approximately 6 months of training, both subjects were producing maximum runs of nine consecutive correct trials and performing at approximately $65 \%$ correct, which was considerably above chance $(16.7 \%)$ on the three successive samples problems.

In the first task shown in Table 1 (1-1), the subject could respond correctly without attending to the sample, thus it only nominally qualifies as a matching task. The 1-2 task is the matching-to-sampie procedure used by most researchers and requires that the subject attend to the sample and be able to distinguish differences in the 10 stimuli for correct matching. All 10 stimuli were used from the beginning of 1-2 training, which may have retarded the animals' learning of the matching concept (Mello, 1971).

Although the 2-2 task may be solved by attending to only one sample (making it logically equivalent to a 1-2 task), our subjects attended to both samples. The monkeys required more trials to acquire the $2-2$ than the $1-2$ task.

Following 2-2 task training, both subjects matched at above-chance levels $(75 \%$ to $85 \%$ correct) with each sample exposed for $300 \mathrm{msec}$. The MSS task with unrestrained monkeys appears to lend itself to studies of presentation time in which sample duration and/or the time between samples (blank time) may be manipulated.

The monkeys experienced considerable difficulty with the 2-3 and 3-3 tasks and had the most difficulty in matching the order of presentation when the samples were from the same category (three colors or three shapes). Over all of the 3-3 training trials, the monkeys were correct on $27 \%$ of the trials when the 
three simples were shapes and on $45 \%$ of the trials when the samples were colors. With combinations of colors and shapes, the subjects were correct $54 \%$ of the total trials.

The notion of greatest confusability of the temporal relationships of samples within a stimulus category was supported by analysis of errors committed on various shape-color combination trials. For example, the monkeys incorrectly matched on $33 \%$ of the trials in which the samples were shape/shape/color. Of the total errors, $61 \%$ were to the incorrect shape as the first response. On color/shape/color trials, $67 \%$ of the errors were first responses to the incorrect color. On combinations of shape/color/color and color/ shape/shape, errors on the second choice were $74 \%$ and $81 \%$, respectively (i.e., the animals selected the first stimulus correctly, but confused the second choice within the same category).

On the tinal 100 trials of the 3-3 task, one animal correctly matched on $70 \%$ of the three color trials, with no better than $50 \%$ correct on the three shape trials. The other monkey matched correctly on $60 \%$ of the three shape trials, but no better than $40 \%$ correct on all color trials.

Following the training reported in Table 1 , one monkey was retrained to $90 \%$ correct performance on the 2-2 and 2-3 tasks. In the $2-3$ retraining task, we eliminated the blue and horizontal line stimuli which seemed confusable with others, increased the separation between successive samples to $300 \mathrm{msec}$, and increased ITI to $30 \mathrm{sec}$ for a correct response and $45 \mathrm{sec}$ for an incorrect response. Wilson, Kaufman, Zieler, and Lieb (1972), using multiple dimensional stimuli in a DMS task, found interference (previous trial intrusions) significant at a $30-\mathrm{sec}$ ITI and mininal at $45 \mathrm{sec}$. Although we did not systematically vary ITI, interference in the initial $2-3$ task was observed. This led us to increase ITI for the 3-3 task and 2-3 task retraining. The increase in blank time, i.e. . time between successive samples, was initiated in the $2-3$ task retraining to make the break between samples more distinctive. The 300 -msec blank time led to an immediate increase in performance. Whether of not long ITI and blank times are necessary cannot be determined from the results of the present study as they were introduced during acquisition of the task without appropriate controls.

An important aspect of the MSS task for studies of memory with animals is the elimination of body-movement cues as delay-interval mediators. In the 3.3 task, there are 720 possible temporal arrangements of three samples from a set of 10 stimuli. If the possible test stimuli arrangements are considered, there are 4,320 possible problems, Also, animals may be trained with certain temporal arrangements and tested over delay intervals with different sample orderings. Other aspects of the MSS task which recommend it for use in the study of animal memory are: (a) control of task variables identitied as important in STM, such as temporal factors (presentation rate and processing time), stimulus variables (associative nonverbal, frequency, and intensity), and, with slight modifications, retention measures (reconstruction, recognition, and conditional matching); and (b) the use of a multiple-unit stimulus, which more closely parallels human STM procedures.

\section{REFERENCES}

D'Amato, M. R. \& O'Neill. W. Effects of delay interval illumination on matching behavior in the capuchin monkey. Journal of the Experimental Analysis of Behavior, 1971, 15, $327-3,33$

JARRARD, L. E.. \& Moise, S. L. Short-term memory in the stumptail $(M$. speciosa): Effects of physical restraint of behavior on performance. Learning and Motivation, 1970, 1, $267-275$.

JaRRARD, L. E., \& Moise, S. L. Short-term memory in the monkey, In L. A. Jarrard (Ed.), Cognitive processes of nonhuman primates. New York: Academic Press, 1971.

Mello, N. K. Alochol effects on delayed matching-to-sample pertormance by rhesus monkey. Physiology and Behavior. 1971. 7. $77-101$

Peterson, L. R., \& Peterson, M. J. Short-term retention of individual items. Journal of Experimental Psychology, 1959, 58, 193-198.

Rorles, F. H. The development of the instrumental skill sequence in the chimpanzee. Journal of the Experimental Analysis of Behavior, 1961, 4, 323-325.

Wilson, M., Kaufman, H. M., Zieler, R. E., \& Lieb, J. P. Visual identification and memory in monkeys with circumscribed inferotem poral lesions. Journal of Comparative and Physiological Psychology, 1972. 78, 173-183.

(Received for putlication December 3, 1974; revision received April 9, 1975.) 